flakes on the bowel towards the middle line and pelvis but in no great quantity. The appendix could not be found at first and a fleshy projection near the caput cæci resembling a stump made it appear possible that it had been removed. However, the impression that this was a mistake remained and further search was made. There was no indication of its presence in the iliac fossa, for the peritoneal surface appeared continuous and there was no undue hardness to be felt. For some time it could not be found but finally towards the pelvis a slight bulging which proved to be the apex of the appendix was discovered and the finger carried along it freed it, with some difficulty, from its bed of adhesions and after ligature of its mesentery it was removed, a second ligature being applied to its proximal end. The stump was not invaginated but its mucous surface was sterilised with a drop of pure carbolic acid. The abdomen was then washed out with gauze sponges in holders and the wound was closed, a glass drain being passed into the pelvis. The appendix was the subject of a chronic catarrhal inflammation and presented a large perforation where a portion of the wall had sloughed which easily admitted the finger-tip. The tube was removed on the third day but discharge continued for some time from the wound. The patient made a satisfactory recovery. I may say that the scar resulting from the former operation is a very good one and without any sign of weakness.

The note of Dr. Mackay, for which I am indebted to him, was to the effect that the patient had had an attack of peritonitis at Christmas time. At the outset appendicitis could not be diagnosed; with the clearing of distension and effusion, however, Dr. Mackay made out a tumour in the right flank which soon showed signs of deep fluctuation. $\mathrm{He}$ suspected peri-appendical suppuration and operated, hoping to find an easily removeable appendix. Some foul pus was evacuated but the appendix could not be found without breaking down the adhesions of the abscess cavity and therefore he had to leave it.

This case is a further confirmation of my contention as put forward in a recent paper that the appendix should be excised in the quiet period, if for some reason it is not removed when an abscess dependent on its disease is opened. Experience of other cases since that statement was written has strengthened my feeling on this question.

We have here a group of four cases which presented symptoms of a mild character varying but slightly. Each patient was seized with abdominal pain and each vomited and had a moderate elevation of temperature-in no case was it registered as above $102^{\circ} \mathrm{F}$. before operation. The pulse did not in any instance (except in Case 4) exceed 110 when I saw it, nor was the expression of any patient indicative of serious abdominal trouble. Yet in each case there was purulent peritoneal exudation of considerable amount within a few hours of the commencement of the illness and in three of them there was perforation of the appendix, and in the fourth acute inflammation of that structure, with a purulent collection inside it. In all these cases the mildness of the symptoms in spite of the serious condition of the appendix is striking. Inflammation of the peritoneum had already commenced and it is unlikely that it would have become localised.

I think that it is very probable that many cases of septic peritonitis have passed through the stage as described in these notes for the patients only to die a few days later because its importance was unrecognised and operative treatment was postponed or neglected. I regard this early effusion as an indication for immediate operation.

Harley-street, W.

\section{THE RELATION OF MENTAL SYMPTOMS TO BODILY DISEASE. ${ }^{1}$}

By NATHAN RAW, M.D. DURH., M.R.C.P. LoND., F.R.S. EDIN.,

PHYSICIAN TO THE MILL ROAD INFIRMARY, LIVERPOOL.

THE relation of mental symptoms to bodily disease is a subject of the greatest importance and interest to us all as practitioners of medicine. It would be impossible for me in one paper to deal with such a comprehensive subject at all adequately, so that I do not propose to speak of

1 A paper read befor the Liverpool Medical Institution on Feb. 13th, actual insanities either as occurring in the course of numerous diseases or as caused by them, but to restrict myself to those temporary mental disturbances observed in association with lesions of various viscera.

Maudsley has said in his "Pathology of Mind" that "it is impossible to be out of sorts physically without being out of sorts mentally," and this we must all admit to be to a great extent true, for our mental well-being depends on the physiological condition of our internal organs, more especially the digestive apparatus, and it is our common experience to observe serious mental disturbance follow viscelal disorder. We are all acquainted with the "livery" man, in what varying moods we may find him at different periodsat one time so irritable as to be approached only with caution and at another so depressed and dull as by preference to be avoided.

In spite of the importance of the subject very little has been written upon it. A few papers dealing with the mental symptoms in individual diseases have been published but as far as I know no general classification or orderly arrangement of them has been made. My own conclusions are based on an observation of a very large number of insane patients in three large county asylums and also about 26,000 ordinary hospital cases. From this very large clinical field of observation I have had exceptional opportunities of seeing almost every form of mental disorder, temporary and permanent, arising during the course of, or following, serious bodily disease and injury.

One of the most interesting forms of mental disorder is that occurring in visceral disease and which has been so well investigated and described by Henry Head in two papersthe first published in Brain in 1893 and the last in the same journal in 1902. The latter excellent and scholarly paper has just been published and I am greatly indebted to it in writing my own paper. Adopting the classification of Head we find that mental changes may be produced in the folloying ways. 1. The circulation to the brain may be so altered either by a disturbed activity of the heart or by the altered condition of the blood. The delirium that accompanies a failing heart may be instanced as an example of a mental change dependent directly on altered vascular conditions. 2. Some secretory organ may be attacked by disease, such as the thyroid. In exophthalmic goitre we have evidences of thyroidism with great nervousness and irritability, whilst in myxodema we have the opposite mental state of depression and hebetude. 3. Disease of excretory organs may lead to the retention of toxic agents in the blood, as, for example, uræmia and jaundice. 4. Poisons circulating in the blood, such as alcohol, may in certain lowered conditions of the body due to visceral disease produce mental changes. 5. "Visceral disease may so lower the patient's resistance that some inherent mental tendency may become manifest as active mental disease." These are the causes of the direct mental effects in visceral diseases and are easily understood, but there are certain diseases of the internal organs which are now known to be accompanied by superficial tenderness of the scalp, the face, and the body, together with radiating pains of the trunk. The pain and tenderness are due to changes in some part of the central sympathetic system caused by disturbance of the internal organ to which sensory sympathetic nerves are supplied. Head calls these "reflected visceral pains" and the mental symptoms are indirect for they are associated with visceral disturbances through the mediation of the sympathetic system. We are all familiar with the young anæmic woman with pain in the chest and side, or even with all sorts of patients who complain of tenderness and radiating pains round the trunk and the head. pains which cause much physical and mental distress and which in many cases are accompanied by changes in consciousness. These changes mostly take the form of hallucinations of sight, hearing, and smell and are generally of a temporary nature, disappearing with the disease, but more pronounced mental changes may manifest themselves in the form of varying moods of exaltation, depression, or suspicion. In these moods a patient may have all the symptoms of an insane person for the time being with delusions, but they are in no sense fixed delusions and often disappear as rapidly as they came. The diseases in which these curious mental states are observed are chiefly those of the heart, the lungs, and the stomach, and it is extraordinary in how many cases they will be found in a greater or lesser degree if carefully looked for. It would be impossible in this short paper to enter at all fully into the details of numerous cases of which I have notes, so that I will 
content myself by describing in a general way the mental state of groups of cases.

In phthisis pulmonalis the mental symptums are often very pronounced and they play an important part in the progress and treatment of the disease. The condition generally known as the "spes phthisica" is not, in my experience, commonly seen and is then seen only in those patients who are not aware of the true nature of their affliction. It is common knowledge that many patients attacked by phthisis suffer very little physical pain and inconvenience - in fact, in very advanced cases except for the cough and expectoration they feel fairly well. It is in these cases where the patient has no idea of the terrible nature of his disease and where a cheerful disposition is preserved that this abnormal hope is described. In my experience the very opposite mental state is often present and in the later stages of phthisis depression, fits of moodiness, with irritability of temper and even delusions of suspicion and fear, are not uncommon. In many cases of phthisis there are intense pain and tenderness over the cervical and dorsal areas with superficial tenderness of the scalp, the forehead, and the neck-reflected visceral pains. In some of these hallucinations of vision are present. A patient will tell you that he has seen a dark figure like a ghost at the foot of his bed in the night which disappears and reappears at intervals. In other cases the patient may develop hallucinations of hearing such as "tapping on the wall," voices from the ceiling, or whispers in his ear. Delusions of suspicion and persecution are sometimes noticed and the patient may think that he is being followed or shadowed and that some impending calamity is upon him. I have observed a few cases in which these delusions have entirely disappeared on the arrest of the phthisis and have not again appeared.

In cardiac disease reflected pain chiefly over the left side of the chest, accompanied by superficial tenderness, is common. This pain is generally over the fifth to the ninth dorsal areas, with tenderness of the forehead, the temples, and the vertex of both sides. The patient may suddenly develop hallucinations of smell and will tell you that the food is tainted and that he has a taste of poison in his mouth and on that account will refuse all food. In other cases there is wild delirium or exaltation of feelings with a sense of great physical strength and anxiety to do hard work.

These mental states are more often present during failing compensation and I have frequently seen them entirely pass away with improvement of the patient's physical condition. All these mental symptoms have up to the present been described and classified as hysteria and hypochondriasis, and it is quite a common thing in making one's visit to the wards for the sister to say that so-and-so has been a little hysterical during the night, perhaps screaming out through fear or saying she has seen ghosts or visions.

I do not pretend for a moment to be able to offer any theory on the association of these curious mental states with reflected visceral pain; that they are frequently present is beyond all doubt and it behoves the physician to be on his guard when they appear lest his patient should be treated either as hysterical or as a lunatic instead of as one who is exhibiting mental symptoms requiring sympathy and care. The conclusions of Head are of interest. Under normal conditions the functions of the viscera are outside consciousness -that is, the heart, the lungs, and the stomach, \&c. carry on their physiological duties in health without our conscious knowledge. "But a complete change takes place when reflected pains of visceral origin come into existence. These sensations produced by abnormal activity of the viscera crowd irto consciousness and usurp the central field of attention. The patient's character appears to be altered. He will become moody, at one time unduly exalted, at another depressed without cause. Reason is displaced and he will have lost control over the expression of his emotions and of his temper." Head concludes his paper in these words: "When the mental states mentioned above appear in consciousness they seem an intrusion from without, an inexplicable obsession that can neither be controlled nor subjected to logical analysis. All such states have since the earliest times been classified as hysteria and I shall be satisfied if I have been'successful in relegating to their proper position a small band of symptoms included under that vast and heterogeneous group."

Heart disease. - I have briefly mentioned before the mental symptoms occasionally seen in heart disease associated with reflected visceral pain, but there is a definite train of mental symptoms often associated with valvular lesions, more espe. cially aortic, and also aneurysms. Some writers describe cardiac insanity as a distinct variety of insanity. These mental symptoms, in my experience, are generally observed towards the close of the disease and are very variable. The patient may develop delusions of suspicion and persecution; he thinks that the uthers in the ward are talking about him and that his food is being poisoned. In heart cases I have known several patients to refuse all medicine on that account. In other cases they are noisy and hilarious with ideas of grandeur and importance. Rarely they are suicidal but several cases are recorded where the patient has thrown himself out of the window or otherwise taken his life. I have for many years been in the habit when lecturing to nurses of impressing upon them the difficulty in nursing the patients in some heart cases. They are especially irritable, suspicious, and ungrateful for all that is done for them and they often take intense dislike to nurses and medical practitioners without any apparent cause. Acute mania sometimes develops in the course of heart disease and I can remember two such patients being sent, to the Portsmouth asylum who rapidly recovered on the improvement of the heart. It is not my intention to speak here of palpitation, tachycardia, or other neuroses of the heart, as they do not fall within the scope of this paper.

Kidney disease. - The relation of the brain and nervous system to the kidneys is very close and at present is little understood, but that the kidneys may be stimulated or suppressed in their functions by purely nervous influences cannot be doubted. The surgeon cuts down upon a kidney or ureter for stone but none is found; the result in some cases is a temporary or permanent suppression of urine. In other cases of suppression of urine from bladder or urethral disease the fact of cutting down on the kidneys and incising them will restore their function. What happens we do not. know. I agree with Dr. C. Savage that there is no special form of insanity associated with kidney disease but in the later stages of chronic Bright's disease the brain seems to participate, although, perhaps, less than we would expect, until it is violently overcome by the uræmic poison. The patients are often found crying and on inquiry they exhibit marked delusions of fear and suspicion; often they are delirious, restless, irritable, and show mental failure until coma terminates the scene preceded by convulsions. As a great clinician observed, "The idle comments foretell the ending of mortality."

It is, however, to the manifestations of uræmia that I wish especially to draw attention. Without any previous warning a patient may be seized with a violent epileptic attack, the fits being of great severity and rapidly passing into the status epilepticus. During pregnancy or when labour is commencing a woman may suddenly become maniacal and may pass into a condition of eclampsia. The mental symptoms here totally overshadow the bodily ones and it is recognised that the patient is in great danger. In both these dangerous conditions total unconsciousness is rapidly reached and general convulsions are the rule. In the gouty contracted kidney, especially in young adults, acute mania is not uncommon and I can remember the cases of three patients who were sent to asylums as lunatics when suffering from this condition. Commencing with restlessness, headache, and insomnia the patient very rapidly passes into a violent state of mania with delusions of fear and suspicion and often ending in death. In another form of uræmia the first symptom noticed is a hemiplegia or partial paralysis with no. brain lesion discoverable post mortem to account for it, and I have seen patients with twitchings, cramps, and hiccough who were quite unable to sleep for several days, the mind remaining quite clear to the end. In some patients the mental distress is pitiable to witness, especially in the dyspnœic form with Cheyne-Stokes respiration or in the condition known as "renal asthma." When a medical man is called to a patient who is irritable, restless, and resistive, with twitchings or even convulsions, or where paralysis or coma is present, he must always have in his mind the possibility of uræmia. It is often impossible to distinguish these cases from cerebral hæmorrhage or poisoning and I have on quite a score of occasions seen cases post mortem whose fracture of the base of the skull or apoplexy turns out to be uræmia and nothing more. I have also had patients sent to hospital and asylum as lunatics who were in the early stages of uræmic poisoning.

Gout.-The exact etiology of gout is unknown but among other theories the nervous one has many adherents. The- 
explosive neuroses and the influence of depressing circumstances, physical or mental, point strongly to the part played by the nervous system in the disease. The extremes of mental action are met with in gout, from maniacal excitement to the most profound melancholia. In acute attacks the patient may be so irritable and bad-tempered as to be unapproachable ; in cases of suppressed or retrocedent gout delirium, apoplexy, or coma may suddenly develop and terminate the disease.

Exophthalmic goitre. - In the interesting and complex disease exophthalmic goitre the nervous and mental symptoms are often the most prominent. In many cases the first symptom observed is a sudden change in the mental condition. The patient becomes abnormally irritable, excitable, and fidgety ; her moral nature, too, is altered and she is untruthful, spiteful, and suspicious. Delusions of impending danger and suspicion are developed and she has all the appearances of a melancholiac. I have seen three such cases sent to asylum who improved immensely under the influence of nerve sedatives and were discharged quite recovered. I have also seen several cases of exophthalmic goitre where the mental symptoms have been absent and the patient has remained placid and good-tempered throughout.

Myxodema. - In myxcedema a condition of mental weakmess is produced by some alteration in the higher intellectual centres, producing a slowness of perception and conduction. It is, however, to be borne in mind that cases of myxcedema may develop acute attacks of insanity, either mania or melancholia with delusions of suspicion and hallucinations of hearing.

Diabetes. - Although there is no known relation between diabetes and insanity we sometimes see definite mental changes coexisting. As a rule a patient is irritable, fretful, and impatient in the earlier stages. In the later stages he becomes suspicious and distrustful of those around him and may even develop delusions. The condition of diabetic coma is too familiar for description and is often most difficult to diagnose until the urine reveals the disease.

Chorea.-We now come to another condition of which we know but little-viz., chorea. We are familiar with its manifestations but not with its cause. I am one of those who think that fright and sudden mental shock play a most important part in its production. I had a patient under my care two years ago who had three distinct attacks of chorea, all caused by a black dog at horae. When this dog was got rid of the patient settled down and has had no further attack. The whole train of mental symptoms in chorea is one of nervous instability and fear. The patient is always in dread of something terrible going to happen and it is only when the medical man and the nurses thoroughly get his confidence that he begins to improve. In the more serious forms of chorea, such as chorea gravidarum, convulsions and coma rapidly ensue and death is common, whilst in a few rare cases insanity follows, and only this week I have sent a boy to the asylum who developed acute mania after an attack of chorea.

Aoute infective fevers. - Amongst the numerous acute infective fevers I will only briefly refer to three-viz, typhoid fever, pneumonia, and rheumatism. In typhoid fever we have two distinct phases of mental disorder, one occurring during the acute febrile stage and the other appearing during convalescence. The nervous symptoms in some cases of typhoid fever completely overshadow all others and the patient may rapidly pass into a condition of raving mania most difficult to treat or to control ; hence the old name for the disease, "nervous fever." The cause of this is toxæmia due to a most severe general infection in which the nervous system participates. In other cases the patient may pass into a condition of wakefulness with hallucinations of vision and hearing and even delusions, which may terminate in a sleep unto death. The acute stage may pass off and the patient during the third week may pass into a state of profound mental prostration, or a condition of imbecility or stupidity may ensue lasting for several weeks. The other important mental change is one of post-typhoid insanity, caused no doubt by nervous exhaustion and insufficient food, and it must be borne in mind that mania may follow ordinary starvation. In some types of typhoid fever insanity not uncommonly follows, but in my experience of a fair number of cases it always passes off and the patient makes a good recovery. I have made a careful observation of the mental changes during an attack of acute pneumonia. In the early stages the symptoms of wild delirium and even mania with all the symptoms of delirium tremens are due no doubt to the virulence of the poison and especially is this the case for some reason or other in apical pneumonia, especially the right apex. I have seen the worst mental symptoms in cases where only a small part of the apex of the lung was attacked and they are very fatal. The sleeplessness so often distressing to witness is due to cerebral irritation at first and then to cerebral exhaustion. It is to be borne in mind that a patient suffering from pneumonia is liable to sudden frenzy and may attack the nurse or patients or may even throw himself out of a window without any warning. I have never observed insanity to follow pneumonia. Mental symptoms in rheumatic fever are extremely rare, not including those, of course, due to hyperpyrexia. I have seen one patient develop acute mania with insanity which terminated in complete recovery after having delusions of grandeur and importance for six weeks.

I will only briefly mention without description the other diseases in which mental symptoms are common. We are all acquainted with alcohol-I mean clinically. It may in excessive doses produce all the symptoms of true insanity, as in addition to the familiar symptoms of delirium tremens delusions of all varieties may be present together with hallucinations of hearing and vision constituting for the time real insanity. Many hundreds of such cases have been sent to asylums and nearly all recover for a time. Arsenic, as we have seen recently, is capable of producing in association with alcohol a curious train of mental symptoms, chiefly depression and melancholia with delusions of fear and suspicion. They pass away slowly but surely and I have only seen two cases out of over 200 in which permanent insanity has remained.

Time will not permit me to speak of the mental symptoms associated with uterine and sexual disorders or to the temporary insanity which sometimes follows injuries and surgical operations, but I would just like to mention the case of a young woman who was sent to the Mill-road Infirmary as a lunatic of three years' standing, who was suffering from delusional insanity and who said that she was the Virgin Mary and was going to give birth to another Christ and so on. She had a large fibroid of the uterus occupying the whole abdomen and extending under the ribs. This was removed and with it the whole of her insanity passed away and she is now perfectly well physically and mentally.

There appears to be a very close relation indeed between the ovaries in the female and the testicles in the male to the nervous system. A few years ago it was a common practice to remove the testicles for diseases of the prostate, especially in elderly men. The operation was followed in several cases by most disastrous results ; acute mania and delusional insanity followed by profound melancholia occurred in three cases under my own observation. The same dire effects follow sometimes in operations on the healthy ovaries and I think that a surgeon should hesitate before removing these organs when in a healthy state for any disease whatever.

I have seen three or four cases of raving acute mania with delusions follow the application of atropine to the eye, always in elderly people, with fortunately a good prognosis.

Now what is the object of this somewhat disjointed paper? There can be no doubt that physicians are now more alive to the fact that the mental symptoms arising in the course of a disease are often part and parcel of it and require just as careful treatment as the disease itself. Too often a patient exhibiting mental symptoms is certified and sent to a lunatic asylum when a little time and patience would have cleared the whole thing up. But, it may be said, where is the alternative? General hospitals and private houses are not equipped for the treatment of mental disease and with regard to the hospitals it is no doubt a grave defect.

There is a great want in Liverpool in the fact that there is no reception house or hospital for those suffering from mental disease and temporary insanity, both from the point of view of the patients and their relatives and the education of students. For instance, a man suffering from acute delirium tremens (and these cases are not uncommon in Liverpool) cannot be admitted anywhere in the city but to the workhouse hospital. These patients are the most dangerous lunatios for the time and yet they cannot be certified as legally insane. In the large Poor-law infirmaries and workhouses if a patient develops temporary insanity he is transferred to the mental wards and there he remains in many instances until he is cured, but in a general hospital or private house he is removed to a lunatic asylum, private or pauper, and is equally well cured there; but the question is, was it necessary for him to bear the stigma of having been signed as a lunatic when he was simply suffering from 
temporary mental aberration the result of bodily disease? There can be no question whatever that an asylum of some sort is the best place for the patient, but he need not be certified as insane, at any rate, until it is seen whether his insanity is likely to be permanent or not. These are the cases which swell up the recovery rate of admissions to asylums, especially those suffering from alcoholic insanity, and $\mathrm{I}$ have always very much doubted the recovery rates from organic insanity. I would like to see established in Liverpool, preferably by the corporation, a reception house for mental diseases of all forms where patients might be admitted in the acute stage of the disease and kept under observation for one month, and at the end of that time, if the symptoms had not passed off, either be removed by their friends or then certified for detention in an asylum.

After a long observation of lunatics-and I have personally certified over 2000 patients to asylums-I am firmly convinced that a large number of people are certified as lunatics throughout the country who are simply suffering from temporary insanity the result of, or associated with, some form of bodily disorder or toxic poisoning. These people are not really insane and should not be associated with lunatics. They quickly recover under proper treatment and I maintain that from every point of view it would be to the public advantage for them to be treated in a hospital for mental diseases, with expert physicians in attendance and a clinique of students to study the acute phases of mental disorder, and where the patient and his relatives might be spared the stigma of his having been detained in a public lunatic asylum as a certified lunatic.

Liverpool.

\section{THE TREATMENT OF EARLY MENTAL CASES IN A GENERAL HOSPITAL.'}

\section{BX J. W. SPRINGTHORPE, M.A., M.D. MELb.,} M.R.C.P. LOND.,

PHySTCIAN TO THE MELBOURNE HOSPITAL; LECTURER ON THERAPEUTICS TO THE UNIVERSITY OF MELBOURNE: OFFICIAL VISITOR TO THE METROPOLITAN ASYLUMS, VICTORIA.

THE classical mistreatment of the lunatic was synchronous with the dominance of the metaphysical conception of the depravity of his volition, and died with its death, perpetuating its cruel existence, however, in the legal definition and procedure that still dare to misjudge man's responsibility by what the lawyers are pleased to call his knowledge. But the modern treatment of the insane, inspired as it is by the modern scientific spirit, is something very different and is ever advancing along lines of progressive reform, so that the phase of development of a community may almost be said to be mirrored by the manner and degree in which it deals with its mentally afflicted.

On looking round we find the following therapeutic details characteristic of the procedure of the most humane and scientific peoples-viz., some provision for early and doubtful cases in the shape of observation wards or receiving houses; the scientific classification of the insane; the separation of the curable in small wards or detached hospitals, furnished with all modern requirements and provided with fullytrained nurses in the proportion of at least one to five; the opening of outdoor departments ; the fusion of hospitals and asylums, and the establishment at the latter of a consultant medical, surgical, and neuro-pathological staff ; the transference of patients from one asylum to another; the licensing of wards in workhouses and the like for the harmless chronics; the boarding-out of increasing numbers by twos and threes in private families, and the official supervision of all private and individual cases. How far we in Victoria fall short of these signs of progress is known only too well to all who take an interest in lunacy matters, and the practical result with us has been that members of the profession here are often at a loss to know how best to deal with numerous individual cases. Too often, also, pauper lunatics enter the asylum through the doorway of the gaol, whilst some of our criminal insane have never got further than the inside of the gaol hospital. It is little wonder therefore that, wherever practicable, the better class are

I A paper read at the April meeting of the Medical Society of Victoria. either treated $s u b$ rosâ in private establishments or sent out of the colony to better quarters abroad.

It was in view of these unfortunate circumstances and their tacit recognition by the authorities that for some years past I have from time to time attempted to treat some of my insane patients in the wards of Melbourne Hospital. The Government provision was so unsatisfactory and inadequate, there was no receiving house, the very names of Kew and Yarra Bend carried an indelible stigma, whilst by hospital treatment there might be for the students in. valuable clinical material that they might otherwise never obtain, and for the patient earlier, and in many respects better, treatment. Most of the advantages of such hospital treatment require simply to be stated to be recognised and admitted:-1. There is no necessity to wait until someunfortunate accident, lâche, or crime necessitates the tardily-written certificate. Treatment is thus available much earlier than usual-a point the importance of which to the patient, to his friends, and to the community can scarcely be over-estimated. 2. It places "borderland " and early cases under the same therapeutic conditions that have been found efficacious against the kindred hysteria, cerebrasthenia, and bodily disease generally, and it gives the patient greater opportunity of having the frequent co-existing or causative bodily disease properly and promptly attended to. 3. It does away with the false distinction between mental and bodily disease and it altogether avoids the stigma and disgrace attaching to "lunatic" and "asylum." 4. There is no signing of certificates with its, at times, serious sequelæ to both patients and medical men, and the natural convalescing ground, the home, is much earlier and more readily available.

These are advantages, both fundamental and immense. But the plan has also its limitations and disadvantages. We had, and have, no cells for separate cases; the nursing staff was, and is, not organised for lunacy work or specially trained in lunacy charge ; whilst the hospital grounds are too small even for ordinary recreation and the public strain upon our beds, their much greater individual cost, and the restric. tions of the law are such as to render it impossible to deal with more than an occasional patient or to keep him under treatment for more than a limited number of weeks. Most, if not all, of these disadvantages, however, seem to me matters of arrangement rather than of principle.

My actual experience may be summarised as follows: 1. Under existing conditions it was found impossible to attempt to deal with maniacal cases. Outside the general wards there was no place for them except the refractory ward and that was fully occupied by the "D.T.'s," the cases of noisy delirium with fever, and the like, and structurally the building itself was quite unsuitable. 2 . We were, however, able to observe and to treat cases of early progressive dementia and of early general paralysis (especially those in which the mental symptoms were late in their development) until we were satisfied that the asylum was the proper place for them. 3. The hospital wards have also been excellent observation wards for many doubtful cases in which it was impossible to say definitely whether the mental condition was or was not the outcome of some undercurrent bodily disease but which, under any hypothesis, required skilled treatment forthwith. Under this heading I have thus dealt with cases of disseminated sclerosis; with mental crises; with cases of syphilitic frontal lepto-meningitis and brain tumours; and with the mental phases of uteroovarian disease, of hypochondriasis, and of doubtful general paralysis. It is beyond question that the critical atmosphere of a hospital medical school and the skilled reports and care of highly-trained sisters are of the greatest value in promoting the diagnosis and treatment of these mental conditions. Other cases, also, complicated with epilepsy, in which the epileptic status was frequent, or unusually severe, or in which the amnesia and dementia were becoming marked, or the onset of actual insanity was suspected, could be kept under observation until it was decided what was best to be done and meantime they could be treated to the best of our ability. 4. Cases of actual lunacy, also, in which the condition was undoubted but the necessity or advisability of signing certificates was more or less problematical, have been satisfactorily treated in the wards. Thus I have treated cases of transient mental unsoundness due to inanition, to. neglect, or concomitant with phosphuria, until the patients have been discharged cured. I have also treated cases of sudden dementia, stupor attonitus, and other diseases, due to shock, fright, and the like, until they, too, have regained 